U. S. EPARTMENT OF
COMMERTE

Natio $Q C$ Bur 100

Standa. $\cup 5753$ No.612 1972 c. 2

\section{Frequency Shifts Due} to Ramsey Type Interrogation in Atomic Beam Tubes 


\section{NATIONAL BUREAU OF STANDARDS}

The National Bureau of Standards ${ }^{1}$ was established by an act of Congress March 3, 1901. The Bureau's overall goal is to strengthen and advance the Nation's science and technology and facilitate their effective application for public benefit. To this end, the Bureau conducts research and provides: (1) a basis for the Nation's physical measurement system, (2) scientific and technological services for industry and government, (3) a technical basis for equity in trade, and (4) technical services to promote public safety. The Bureau consists of the Institute for Basic Standards, the Institute for Materials Research, the Institute for Applied Technology, the Center for Computer Sciences and Technology, and the Office for Information Programs.

THE INSTITUTE FOR BASIC STANDARDS provides the central basis within the United States of a complete and consistent system of physical measurement; coordinates that system with measurement systems of other nations; and furnishes essential services leading to accurate and uniform physical measurements throughout the Nation's scientific community, industry, and commerce. The Institute consists of a Center for Radiation Research, an Office of Measurement Services and the following divisions:

Applied Mathematics-Electricity-Heat-Mechanics-Optical Physics-Linac Radiation ${ }^{2}$ - Nuclear Radiation ${ }^{2}$ - Applied Radiation ${ }^{2}$ - Quantum Electronics ${ }^{3}$ Electromagnetics ${ }^{3}$ - Time and Frequency ${ }^{3}$ - Laboratory Astrophysics ${ }^{3}$ - Cryogenics ${ }^{3}$.

THE INSTITUTE FOR MATERIALS RESEARCH conducts materials research leading to improved methods of measurement, standards, and data on the properties of well-characterized materials needed by industry, commerce, educational institution's, and Government; provides advisory and research services to other Government agencies; and develops, produces, and distributes standard reference materials. The Institute consists of the Office of Standard Reference Materials and the following divisions:

Analytical Chemistry-Polymers-Metallurgy-Inorganic Materials-Reactor Radiation-Physical Chemistry.

THE INSTITUTE FOR APPLIED TECHNOLOGY provides technical services to promote the use of available technology and to facilitate technological innovation in industry and Government; cooperates with public and private organizations leading to the development of technological standards (including mandatory safety standards), codes and methods of test; and provides technical advice and services to Government agencies upon request. The Institute also monitors NBS engineering standards activities and provides liaison between NBS and national and international engineering standards bodies. The Institute consists of the following technical divisions and offices:

Engineering Standards Services-Weights and Measures-Flammable FabricsInvention and Innovation-Vehicle Systems Research-Product Evaluation Technology-Building Research-Electronic Technology-Technical AnalysisMeasurement Engineering.

THE CENTER FOR COMPUTER SCIENCES AND TECHNOLOGY conducts research and provides technical services designed to aid Government agencies in improving cost effectiveness in the conduct of their programs through the selection, acquisition, and effective utilization of automatic data processing equipment; and serves as the principal focus within the executive branch for the development of Federal standards for automatic data processing equipment, techniques, and computer languages. The Center consists of the following offices and divisions:

Information Processing Standards-Computer Information-Computer Services - Systems Development-Information Processing Technology.

THE OFFICE FOR INFORMATION PROGRAMS promotes optimum dissemination and accessibility of scientific information generated within NBS and other agencies of the Federal Government; promotes the development of the National Standard Reference Data System and a system of information analysis centers dealing with the broader aspects of the National Measurement System; provides appropriate services to ensure that the NBS staff has optimum accessibility to the scientific information of the world, and directs the public information activities of the Bureau. The Office consists of the following organizational units:

Office of Standard Reference Data-Office of Technical Information and

Publications-Library-Office of Public Information-Office of International Relations.

\footnotetext{
${ }^{1}$ Headquarters and Laboratories at Gaithersburg, Maryland, unless otherwise noted; mailing address Washington, D.C. 20234.

2 Part of the Center for Radiation Research.
}

${ }^{3}$ Located at Boulder, Colorado 80302. 
MAR 21972 -not oCe

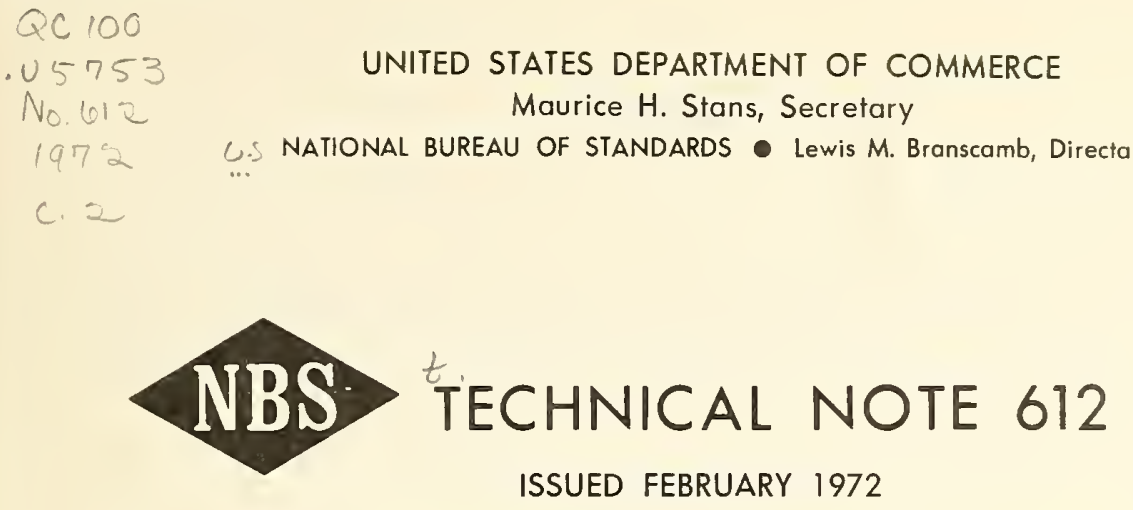

Nat. Bur. Stand. (U.S.), Tech. Nate 612, 25 pages (February 1972)

CODEN: NBTNA

\title{
Frequency Shifts Due to Ramsey Type Inferrogation in Atomic Beam Tubes
}

\author{
Helmut Hellwig, J. A. Barnes, and D. J. Glaze \\ Time and Frequency Division \\ Institute for Basic Standards \\ National Bureau of Standards \\ Boulder, Colorado 80302 \\ and \\ Peter Kartaschoff \\ Laboratoire Suisse de Recherches Horlogères \\ Neuchâtel, Switzerland
}

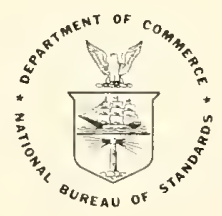

NBS Technical Nates are designed ta supplement the Bureau's regular publicatians pragram. They pravide a means far making available scientific data that are af transient ar limited interest. Technical Nates may be listed ar referred to in the apen literature. 


\section{FOREWORD}

This Technical Note consists of two related papers on the topic of Frequency Shifts Due to Ramsey Type Interrogation in Atomic Beam Tubes. The first paper (Part I) was presented at the 25th Annual Symposium on Frequency Control, Fort Monmouth, N.J., April 1971; the second one (Part II) at the IEEE Eurocon, Lausanne, Switzerland, October 1971. This Technical Note may, therefore, be considered to be a documentation of these talks.

Helmut Hellwig, J. A. Barnes, and D. J. Glaze are with the National Bureau of Standards. Peter Kartaschoff was a National Bureau of Standards' Visiting Scientist from October 1970 to September 1971; he is with the Laboratoire Suisse de Recherche Horlogères, Neuchâtel, Switzerland. 


\section{TABLE OF CONTENTS}

$\underline{\text { Page }}$

Part I: Frequency Biases in a Beam Tube Caused by

Ramsey Excitation Phase Differences. . . . . . . . . 1

by Helmut Hellwig, J. A. Barnes, and D. J. Glaze

1. Introduction . . . . . . . . . . . . . 2

2. Calculation of Frequency Shifts... . . . . . 2

3. Frequency Modulation Amplitude Dependence . . . 5

4. Power Dependence. . . . . . . . . . . 6

5. Experimental Results . . . . . . . . . 6

6. Conclusions . . . . . . . . . . . 8

Part II: Power-Linewidth Relations in Atomic Beam Tubes . . . . 14 by Peter Kartaschoff

1. Introduction . . . . . . . . . . . 14

2. Single Velocity Power-Linewidth Relation . . . . 15

3. Conclusions . . . . . . . . . . . 18 



\title{
PART I:
}

\section{FREQUENCY BIASES IN A BEAM TUBE}

\section{CAUSED BY RAMSEY EXCITATION PHASE DIFFERENCES}

\author{
Helmut Hellwig, J. A. Barnes, and D. J. Glaze
}

A phase difference between the two interaction regions of a Ramsey excitation resonance structure results in a frequency bias in the measured beam resonance. A simple mathematical model is discussed which describes the dependence of this bias on the phase difference, the microwave power level, the modulation amplitude, and the resonance linewidth. This dependence results from the interaction of the modulated microwave excitation frequency with the asymmetric shape of the slightly shifted resonance line. In a first order approximation, no dependency on the frequency modulation amplitude is expected. Near-linear dependencies on the linewidth and microwave power level which are quite pronounced even at relatively small cavity phase differences are predicted.

The theoretical results are compared with one set of experimental data on the microwave power dependence as measured in 1969 with the cesium beam standard NBS-III. After a correction is applied to remove the power dependence due to spectral impurity of the microwave excitation, the remaining measured power dependence agrees quantitatively with that calculated using a cavity phase difference of about 3 milliradians.

Key words: Cavity phase shift; cesium beam tube; frequency accuracy; frequency standard; power shift; resonance line shape. 


\section{INTRODUCTION}

In accordance with the international definition [1], the unit of time interval, the second, is realized with a cesium beam apparatus. Several bias corrections have to be applied to the measured resonance frequency in order to obtain the resonance frequency of the unperturbed, free cesium atom [2]. In this paper we consider only those bias corrections which are associated with a phase difference between the two interaction regions of a Ramsey excitation resonance structure [3].

In this paper we present a simple mathematical model which allows analytical solutions to the question of frequency shifts in the presence of a cavity phase difference. The results are intended to aid in the understanding of the physical mechanisms involved. Although our mathematical model is only an approximation to the physical situation, and is tailored to a particular set of conditions, we feel that it is close enough to the real conditions which we re encountered in the National Bureau of Standards Frequency Standard [2], NBS-III, to warrant a quantitative comparison with experimental data from this apparatus. This is done in Section 5 of this paper.

\section{CALCULATION OF FREQUENCY SHIFTS}

We proceed on the basis of the following set of assumptions and approximations: (a) No frequency deviations from resonance exceed the linewidth; (b) the microwave interrogation power does not deviate greatly from optimum power; ${ }^{1}$ (c) the modulation of the microwave signal is sinusoidal; (d) the modulation frequency is less than the resonance linewidth; (e) the velocity distribution in the atomic beam is Maxwellian; and (f) $\ell<<L$ where $\ell$ and $L$ are the lengths of the interaction and drift regions, respectively, as defined by Ramsey [3].

In the presence of a cavity phase difference the central peak of the Ramsey resonance pattern is displaced from the resonance frequency I Optimum power is that microwave power which leads to a maximum in the detected beam intensity. 
of the unperturbed atom. Furthermore, the lineshape displays a certain asymmetry. We restrict ourselves to the conditions (a) and (b) above and can in first order approximate the central peak of the Ramsey pattern, I $\{\nu\}$, by

$$
I\{\nu\}=A+B \varphi \sin \left\{\frac{\pi\left(\nu-\nu_{\varphi}\right)}{\mathrm{U}}\right\}+\mathrm{C} \cos \left\{\frac{\pi\left(\nu-\nu_{\varphi}\right)}{\mathrm{W}}\right\} .
$$

In eq (I-1) $A, B$, and $C$ are constants under given experimental conditions; $\mathrm{W}$ is the linewidth at half intensity; $U$ has the characteristic of a linewidth, $U \equiv W$ for monovelocity atoms, $U$ is the same order of magnitude as $W$ for our assumption (e) above; and $\nu_{\varphi}$ is a parameter related to the cavity phase difference $\varphi$. Using condition (e) above, it can be shown that

$$
\nu_{\varphi}-\nu_{0}=\frac{3 \varphi}{4 \pi} \mathrm{W}
$$

In eq $(I-2) \nu_{0}$ is the resonance frequency in the absence of a cavity phase difference. Because of the asymmetry, the frequency of the central peak $\nu_{\mathrm{p}}$ differs from $\nu_{\varphi}$. By setting $\mathrm{dI}\{\nu\} / \mathrm{d} \nu=0$, we obtain

$$
\nu_{p}=\nu_{\varphi}+\frac{B W^{2} \varphi}{\pi C U}
$$

We now proceed to calculate the frequency to which the slave oscillator frequency servo will "tune-in." If we modulate $\nu$ in a sinusoidal fashion [condition (c) above] about some value $\nu_{1}$ with the amplitude $\nu_{\mathrm{m}}$ and the angular frequency $\omega$ we can write

$$
\nu\{t\}=\nu_{1}+\nu_{m} \sin \omega t .
$$

We now make use of as sumption (d) above and substitute eq (I-4) into eq (I-1), using eq (I-3) to express B. We obtain

$$
\begin{aligned}
& I\{t\}=A+C\left[\left(\nu_{p}-\nu_{\zeta}\right) \frac{\pi U}{W^{2}} \sin \right.\left\{\frac{\pi}{U}\left(\nu_{1}-\nu_{\varphi}+\nu_{m} \sin \omega t\right)\right\} \\
&\left.+\cos \left\{\frac{\pi}{W}\left(\nu_{1}-\nu_{\varphi}+\nu_{m} \sin \omega t\right)\right\}\right] .
\end{aligned}
$$


We are interested in the coefficient of that term in $I\{t\}$ which varies as sin $\omega t$, because the servo forces this term to be zero.

Now

$$
\begin{aligned}
& \sin \left\{\frac{\pi}{U}\left(\nu_{1}-\nu_{\varphi}+v_{m} \sin \omega t\right)\right\} \\
& =2 \cos \left\{\frac{\pi}{U}\left(\nu_{1}-v_{\varphi}\right)\right\} J_{I}\left\{\frac{\pi}{U}\right\} \sin \omega t+\text { harmonics }
\end{aligned}
$$

and

$$
\begin{aligned}
& \cos \left\{\frac{\pi}{W}\left(\nu_{1}-\nu_{\varphi}+\nu_{m} \sin \omega t\right)\right\} \\
& =-2 \sin \left\{\frac{\pi}{W}\left(\nu_{1}-\nu_{\varphi}\right)\right\} J_{1}\left\{\frac{\pi \nu_{m}}{W}\right\} \sin \omega t+\text { harmonics. }
\end{aligned}
$$

Then the coefficient of $\sin \omega t$ in $I\{t\}$ [eq (I-5)] vanishes when

$$
\begin{aligned}
& \sin \frac{\pi}{W}\left(\nu_{1}-\nu_{\varphi}\right) \\
& =\left(\nu_{p}-\nu_{\varphi}\right) \frac{\pi U}{W^{c}} \cos \frac{\pi}{U}\left(\nu_{1}-\nu_{\varphi}\right) \frac{J_{1}\left(\frac{\pi \nu^{m}}{U}\right)}{J_{1}\left(\frac{\pi \nu_{m}}{W}\right)} .
\end{aligned}
$$

$\nu$ is the frequency to which the servo will "tune-in", and thus the 1

resonance frequency of the beam tube. Note that $v_{1}$ is not equal to either $\nu_{\mathrm{p}}$ or $\nu_{\varphi}$

We have assumed that the off set is always small compared to the linewidth, i.e., $\left(\nu_{1}-\nu_{\varphi}\right)<<W,\left(\nu_{1}-\nu_{\varphi}\right)<U$, and we can rewrite eq (I-6)

$$
\nu_{1}-\nu_{\varphi} \approx\left(\nu_{p}-\nu_{\varphi}\right) \frac{U_{1}\left\{\frac{\pi}{U} \nu_{m}\right\}}{W J_{1}\left\{\frac{\pi}{W} \nu_{m}\right\}} \text {. }
$$

Substituting eq (I-2) and eq (I-3) into eq (I-7) we obtain

$$
\nu_{I}-\nu_{0} \approx \frac{3 \varphi \mathrm{W}}{4 \pi}\left[1+\frac{4 \mathrm{~B}}{3 \mathrm{C}} \frac{\mathrm{J}_{1}\left\{\frac{\pi}{\mathrm{U}} \nu_{\mathrm{m}}\right\}}{\mathrm{J}_{1}\left\{\frac{\pi}{\mathrm{W}} \nu_{\mathrm{m}}\right\}}\right] .
$$


Equation (1-8) gives us the frequency bias $\Delta \nu$ which we define

$$
\nu_{1}-\nu_{0} \equiv \Delta \nu
$$

In order to evaluate eq(I-8) we have to express B/C in terms of the operating conditions. Following eq(I-1)we choose a linear approximation ba sed on the numerical results of [3], and we obtain $\mathrm{B} \varphi \pi / \mathrm{C} U \approx 2 \varphi / 3 \pi \mathrm{W}$. $W_{0}$ is the linewidth at optimum power $P_{0}$. Equation $(I-8)$ can now be written

$$
\Delta \nu \approx \frac{30 \mathrm{~W}}{4 \pi}\left[1+\frac{8 \mathrm{U} \mathrm{J}_{1}\left\{\frac{\pi}{\mathrm{U}} \nu_{\mathrm{m}}\right\}}{9 \pi^{2} \mathrm{~W}_{\mathrm{O}} \mathrm{I}_{1}\left\{\frac{\pi}{\mathrm{W}} \nu_{\mathrm{m}}\right\}}\right] \text {. }
$$

\section{FREQUENCY MODULATION AMPLITUDE DEPENDENCE}

In eq(I-10) any dependence on the frequency modulation amplitude is solely due to the arguments of the Bessel functions which contain $\nu_{m}$. The approximate nature of our discussion requires that we restrict our discussion to modulation amplitudes not exceeding the optimum modula tion, that is to

$$
\nu_{\mathrm{m}} \leq \nu_{\mathrm{mo}} \text { where } \nu_{\mathrm{mo}} \equiv \frac{1}{2} \mathrm{~W}_{\mathrm{o}} .
$$

This and the fact that $U$ and $W$ can be expected not to differ greatly leads us in a first order approximation to

$$
\Delta \nu \approx \frac{3 \varphi \mathrm{W}}{4 \pi}\left[1+\frac{8 \mathrm{~W}}{9 \pi^{2} \mathrm{~W}_{\mathrm{O}}}\right]
$$

According to eq ( $1-12) \Delta v$ has no functional dependence on $\nu_{\mathrm{m}}$. We conclude that (in the limits of our approximation) no frequency modula tion amplitude dependent frequency bias is to be expected for $\nu_{\mathrm{m}} \leq \nu_{\mathrm{mo}}$. This result is in accordance with our experimental observations on the cesium beam frequency standards at the National Bureau of Standards. 


\section{POWER DEPENDENCE}

We can use eq (I-12) to obtain the dependence of the frequency bias $\Delta \nu$ on the microwave power P. Equation (I-12) shows that the frequency bias is a function of the phase difference and the resonance linewidth. The linewidth, moreover, is a function of the microwave power level. This can be understood intuitively if one realizes that with decreasing microwave power the slower molecules become relatively more effective which results in a corresponding line-narrowing [3]. The frequency bias $\Delta v$ thus becomes power dependent. A numerical calculation of the relationship between linewidth and microwave power is depicted in figure 1. In figure 2 we depict the frequency bias $\Delta \nu$ as a function of both linewidth [eq (I-12)] and microwave power [eq (I-12) and fig. 1]. The ordinate is normalized to $\Delta \nu / \Delta \nu_{0}=1$ at optimum power, $\mathrm{P}=\mathrm{P}_{\mathrm{O}}$ and $\mathrm{W}=\mathrm{W}_{\mathrm{O}}$, where $\Delta \nu_{0}$ denotes the frequency bias at optimum power.

Equation (I-12) and figure 2 show that the power dependence of the frequency bias is almost linear; however, we note that the extrapolation of this near-linear portion to $\mathrm{P} \rightarrow 0$ does not yield a vanishing frequency bias. A reduction of power from $P_{0}$ to $P_{0} / 2$ will result in a frequency bias change of about $1 / 3$ of the total bias at optimum power or more accurately

$$
\Delta \nu\left\{\mathrm{P}_{0} / 2\right\} \approx 0.70 \Delta \nu_{0}
$$

\section{EXPERIMENTAL RESULTS}

The reported measurements were performed during the accuracy evaluation of the cesium beam frequency standard, NBS-III, in 1969 [2]. Figure 3 depicts the experimental results. The solid lines are a plot of the fraction frequency change as a function of the microwave power level for the two opposite beam directions. The precision of these measurements is discussed in [2]. The microwave power changes ranged from $P$ (optimum power) to not less than one-fourth $P_{0}$. The actual value for $P_{0}$ was 
$P_{0}=1.4 \mathrm{~mW}$; the frequency modulation amplitude was adjusted to $\nu_{\mathrm{m}} \approx \nu_{\mathrm{mo}}$. The two slopes are $+22 \times 10^{-13}$ per $\mathrm{mW}$ and $-11 \times 10^{-13}$ per $\mathrm{mW}$. The total fractional frequency change with beam reversal at $P=P_{0}$ is $78 \times 10^{-13}$ which gives us $\Delta \nu_{0} / \nu_{0}=39 \times 10^{-13}$.

We believe that the asymmetry in the two slopes of figure 3 is caused by asymmetries in the spectrum of the microwave signal. ${ }^{2}$ Such effects may be expected to be unchanged under beam reversal and to depend linearly on the microwave power (for a small single sideband perturbation) [3]. We obtain symmetric slopes if we subtract a slope of $+5.5 \times 10^{-13}$ per $\mathrm{mW}$. Thus we can conclude that the spectrum causes a power shift of $+5.5 \times 10^{-13}$ per $\mathrm{mW}$ (dashed line in fig. 3), and the cavity phase shift a power dependence of $\pm 16.5 \times 10^{-13}$ per $\mathrm{mW}$ (dashed-dotted lines in fig. 3).

As we expected theoretically, we have a seemingly linear functional dependence. The measured biases at optimum power and half-optimum power are respectively, $\Delta \nu_{0} / \nu_{0}=39 \times 10^{-13}$ and $\Delta \nu\left\{P_{0} / 2\right\} / \nu_{0}=27.5 \times 10^{-13}$ or

$$
\Delta \nu\left\{\mathrm{P}_{\mathrm{O}} / 2\right\} \approx 0.71 \Delta \nu_{\mathrm{O}}
$$

which is in agreement with the theoretical results of figure 2 and eq (I-13).

We can now calculate the cavity phase shift $\varphi$ from eq $(I-12)$. The numerical calculation yields

$$
\Delta \nu_{0}=0.26 \mathrm{~W}_{0} \varphi
$$

The linewidth was measured to be $W_{0}=45 \mathrm{~Hz}$ and we obtain from eq (I-15) with the power dependent frequency change discussed before $\varphi \approx 3 \times 10^{-3}$ radians.

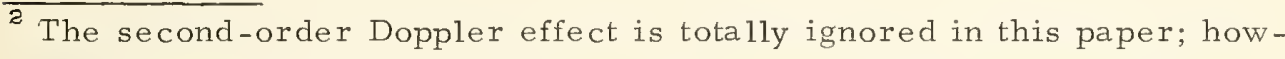
ever, it must be taken into consideration when extreme accuracies are the objective. A. G Mungall recently treated this problem [4].
} 


\section{CONCLUSIONS}

We presented an analytical discussion of frequency shifts in a beam tube which relate to the presence of a cavity phase difference between the two Ramsey excitation regions. The results are approximate and may be expected to be different for different experimental conditions (see Section 2). ${ }^{3}$

The results, as summarized in eqs (I-10) and (I-12) and in figure 2, show that frequency shifts caused by cavity phase differences, the microwave power level, the resonance linewidth (velocity of atoms), and the frequency modulation amplitude are closely interrelated.

Frequency changes due to changes in the microwave power level are quite pronounced, which is contrary to the result of a previous treatment of this subject [5]. In fact, the power dependence could be utilized to determine the frequency bias when other causes for frequency bias are known to be absent, e.g., microwave spectrum effects (see Section 5). In this case the measurement of the frequency change with reduction of the microwave power to, for example, $\mathrm{P}_{0} / 2$ yields directly the frequency bias according to eq (I-13). We have used this method in recent experiments on new cesium beam tubes.

$\overline{3}$ The treatment of the power dependence due to cavity phase differences, as presented in this paper, leads to a rather simple physical picture: The chosen microwave power level acts like a velocity selector because only atoms within a rather narrow velocity range will have a significant transition probability. Atoms with velocities other than that selected by the given power level do contribute, but only in some minor fashion as may be seen from eq (I-12) and figures 1 and 2 . 
A cavity phase difference will, in general, also cause frequency shifts to occur if the frequency modulation amplitude is changed. The frequency changes are virtually absent if a frequency modulation amplitude of less than half the linewidth is chosen (Section 3). However, if larger frequency modulation amplitudes are used or if the cavity phase difference is unusually large (large bias) this effect could become significant. An analytical treatment which is valid for these conditions would require a higher order approximation than was attempted in this paper.

In the presence of a spectrum-related frequency bias of unknown magnitude, methods like the one discussed above will not be adequate. Beam reversal presently appears to be the only method ${ }^{4}$ which then allows the separation and individual measurement of the different frequency biases. Beam reversal changes the sign in eq (I-12); consequently, one should then obtain microwave power (and frequency modulation amplitude) dependencies which are identical for the two beam directions, except for the sign reversal. Any deviations from this symmetry would indicate the presence of additional effects, e.g., signal spectrum asymmetry. The frequency biases can then be obtained as was demonstrated in Section 5 (fig. 3).

We summarize: In the absence of other biases, e.g., spectrumrelated biases, variation of the microwave power offers a way to easily detect and correct for a cavity phase difference and the related frequency bias. This method requires a rather broad velocity distribution, e.g., a Maxwellian, and would not lead to any useful results in the limit of a monovelocity beam.

Beam velocity changes [6], as applied to the detection and correction of cavity, phase differences, act in a way quite similar to the variation of the microwave power. Both act on $W$ in eq(I-12). However, this method also should only be used with great caution if spectrum-related frequency biases are present. 


\section{ACKNOWLEDGMENT}

We thank Dr. Stephen Jarvis, Jr. for his assistance in computing the data of figure 1 and for his very valuable, constructive criticism. 


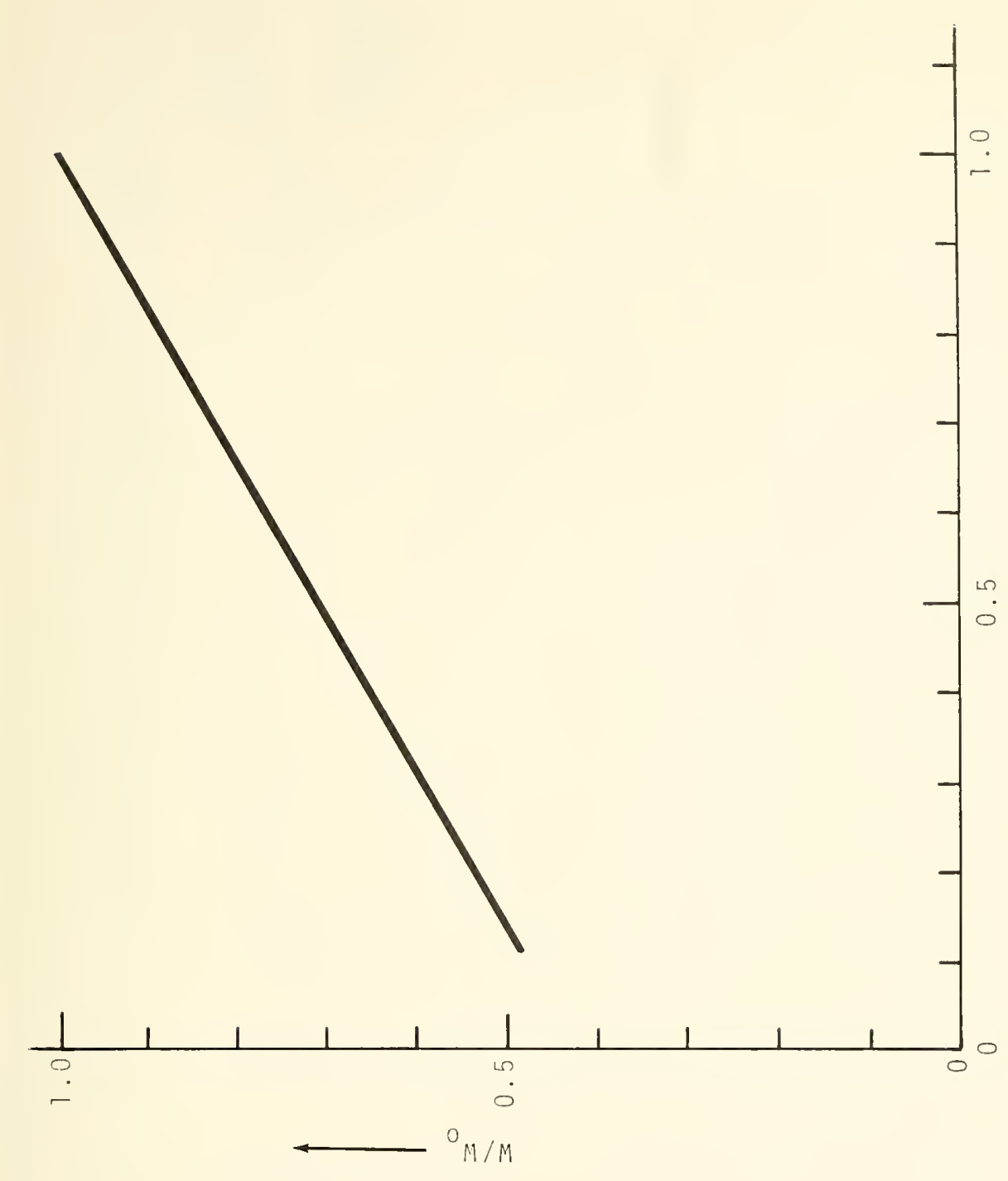




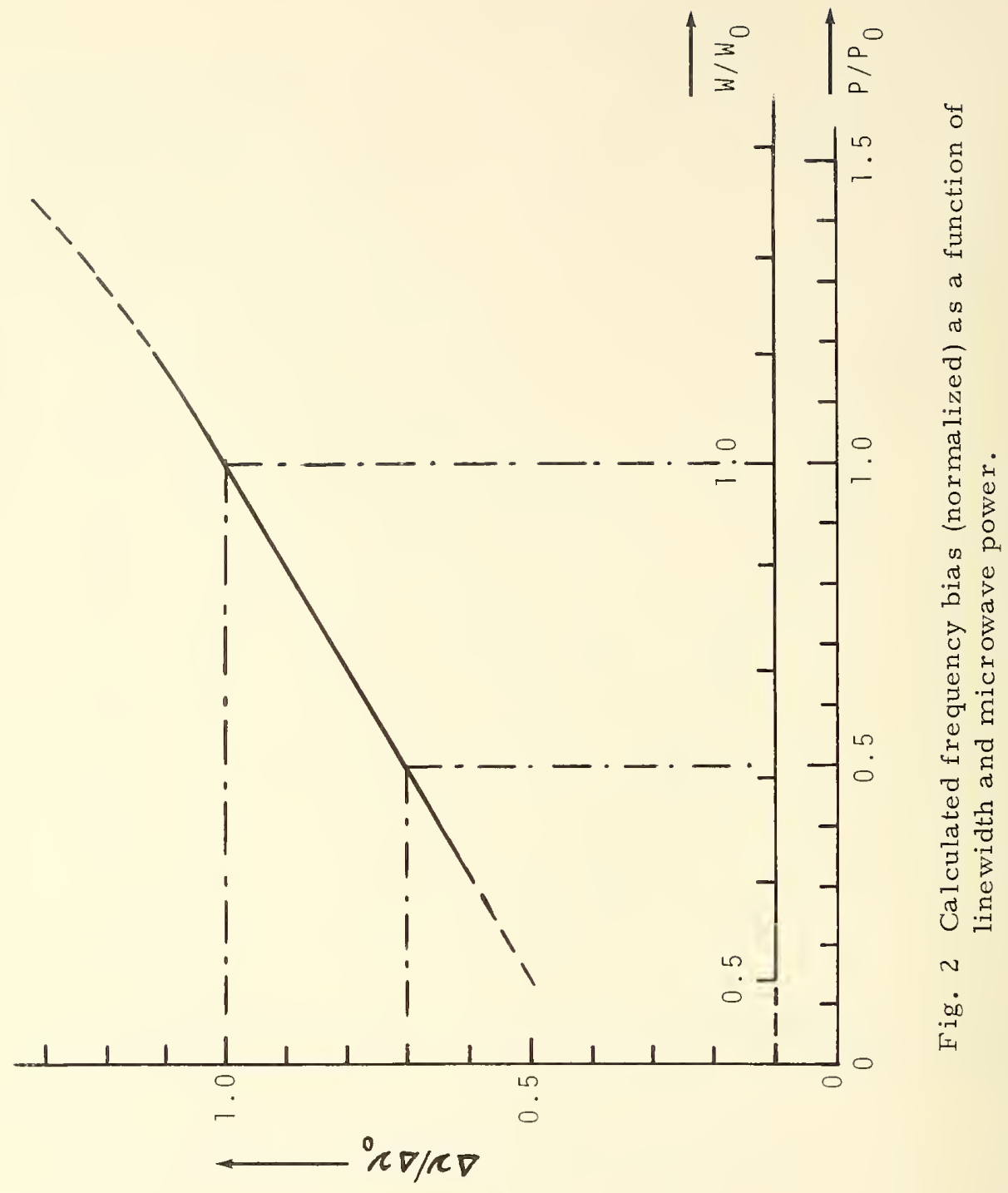




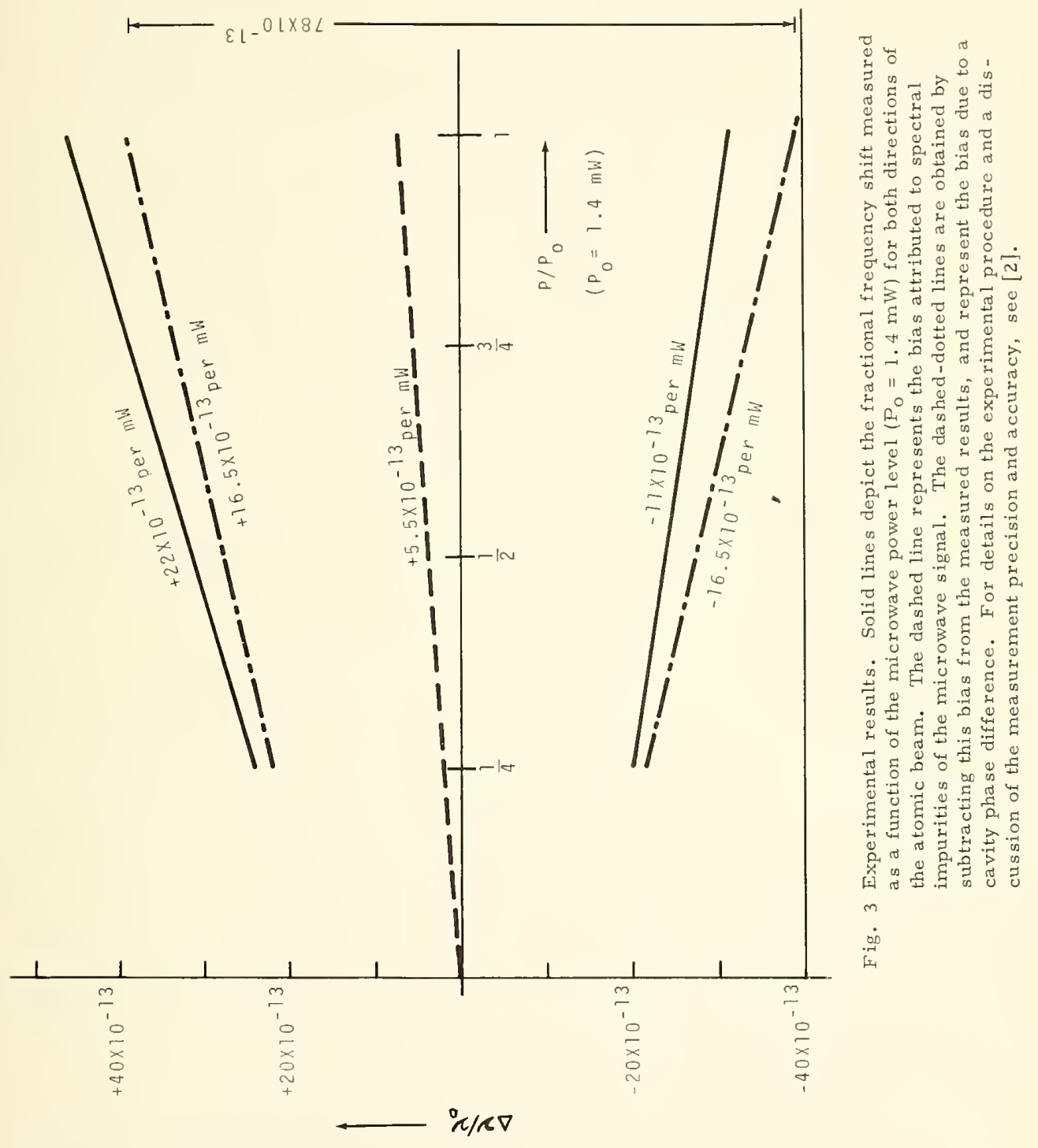




\section{POWER-LINEWIDTH RELATIONS IN ATOMIC BEAM TUBES}

\section{Peter Kartaschoff}

One of the most serious effects limiting the accuracy of atomic beam resonators is the uncertainty of phase shifts between the oscillating fields in a Ramsey excitation cavity. A technique using the linewidth dependence on the excitation power to measure the phase-shift induced bias has recently been proposed. The conditions under which this method is valid and their implications on the design of beam optics will be discussed with consideration of available experimental results.

Key words: Cavity phase shift; cesium beam tube; frequency accuracy; frequency standard; power shift; resonance line shape.

\section{INTRODUCTION}

One of the most serious effects limiting the accuracy of atomic beam resonators is the uncertainty of the phase difference between the two separated oscillating fields in a Ramsey cavity. A technique for measuring the frequency bias caused by this phase difference has been described by H. Hellwig, J. A. Barnes, and D. J. Glaze (Part I). This technique is based on the dependence of the resonance linewidth on the applied microwave power, which results in a power-dependent frequency shift if any phase difference is present. In this part, a different theoretical approach based on an approximation to the transition probability for singlevelocity atoms leads to a simple relation between excitation power and linewidth which is valid for any sufficiently broad, but not necessarily Maxwellian, velocity distribution. 


\section{SINGLE VELOCITY POWER-LINEWIDTH RELATION}

The theoretical considerations presented in Part I are valid only for a resonator where the full modified Maxwellian velocity as defined in ([3], eq II-26) is present at the detector. In many cases, however, especially in commercial-type beam tubes and in designs using multipole state-selectors, the velocity distribution of the detected atoms is severely restricted by the beam optics. This appears to be the result of ray-tracing optimizations attempting to maximize the "figure of merit" which is proportional to the ratio between the square root of the resonance amplitude and the linewidth.

In particular, a sharp cutoff in the velocity distribution is observed below the optimum velocity selected by the state selectors. In these cases, no significant narrowing of the linewidth is observed if the microwave excitation is reduced below the optimum value $\mathrm{P}_{0}$.

On the other hand, there remains the possibility to broaden the linewidth by increasing the power beyond the optimum value provided that faster atoms can still be detected. Pending experimental verification, this technique appearsfeasible if the microwave signal is free from undesired sidebands.

The bias due to second order Doppler shift also depends on the velocity distribution and thus on the applied microwave power. This problem has recently been treated by A. G. Mungall [4].

For single velocity atoms, the transition probability given by Ramsey ([3], eq V-44) can be approximated near resonance in the following way, leading to his eq $(V-44 a)$ :

$$
\mathrm{P}_{\mathrm{pq}}=\frac{\mathrm{I}}{\mathrm{I}_{\max }}=\frac{1}{2} \sin ^{2} 2 \mathrm{~b} \tau[1+\cos (\lambda \mathrm{T}+\varphi)],
$$

using Ramsey's notation, i.e., 
b is the perturbation amplitude in $s^{-1}$, as defined in [3];

$\tau=\frac{\ell}{\mathrm{V}}$ the time of flight through each cavity;

$\mathrm{T}=\frac{\mathrm{L}}{\mathrm{V}}$ the time of flight through the drift region between the 2 cavities;

$0=$ the phase difference in radians between the oscillating fields;

$\lambda=\omega_{0}-\omega$ the angular frequency detuning, $\omega_{0}=2 \pi \nu_{0}$.

Note that I have changed the sign of $\varphi$ with respect to that used by Ramsey, i.e., the first field is assumed leading the second. This has been done in order to remain consistent with Part I, where the same situation has been implicitly as sumed.

Equation (II-1) is valid under the following as sumptions:

$$
2 b>>|\lambda|
$$

is the "near resonance condition" as stated by Ramsey.

It can be easily shown that this condition remains valid for a detuning larger than $W / 2$ and also for a wide power range with a lower limit of about $0.3 \mathrm{P}_{0}$ with $l \approx 1 \mathrm{~cm}$. The upper limit of applied power is only given by the validity of the perturbation method used in calculating the probability distribution and is thus beyond our considerations, since it requires $b<<\omega_{0}$. We now compare eq (II-I) with eq (I-I). The linewidth being equal to $W=\frac{1}{T}$, the argument of the cosine term is

$$
\lambda T+\varphi=\frac{\pi}{W}\left(\nu_{0}-\nu\right)+\varphi
$$

and with $\varphi<<1$, we can write

$$
\mathrm{P}_{\mathrm{pq}}=\frac{1}{2} \sin ^{2} 2 \mathrm{~b} \tau\left(1+\varphi \sin \frac{\pi}{W}\left(\nu-\nu_{\mathrm{o}}\right)+\cos \frac{\pi}{W}\left(\nu-\nu_{\mathrm{o}}\right) .\right.
$$

Equation (I-1) reduces to the same form by setting there:

$$
\begin{aligned}
& \mathrm{A}=\mathrm{B}=\mathrm{C}=\frac{\mathrm{I}_{\max }}{2} \sin ^{2} 2 \mathrm{~b} \tau \\
& \mathrm{U}=\mathrm{W} \text { and } \nu_{\varphi}=\nu_{0} .
\end{aligned}
$$


The peak frequency $(\mathrm{d} I / \mathrm{d} \nu=0)$ is then given by

$$
\nu_{\mathrm{p}}-\nu_{\mathrm{o}}=\frac{\mathrm{W \omega}}{\pi} \text {. }
$$

The "near resonance" condition assumed above replaces conditions (a), (b), and ( $f$ ) and is less restrictive. Conditions (c) and (d) remain unchanged insofar as sinusoidal modulation is considered. (See Part I, Section 2.)

Condition (e) has no meaning here since we start from a single velocity approximation. From any known velocity distribution including the full modified Maxwellian, averaging and weighting can easily be done on a computer.

On the other hand, it is not possible to draw conclusions for a non-Maxwellian distribution from results assuming a full Maxwellian distribution. The usefulness of the latter assumption is the refore rather restricted. We therefore replace condition (e) by the following assumption: The velocity distribution function is assumed to be broad and to have bounded first and higher derivatives.

In other words, this means that we should be able to detect a significant number of atoms over a wide range, e.g., 2:1 velocity range, with intensity variations of not more than $2: 1$ over that range. Then we may consider the velocity selection being performed by the microwave power according to the factor $\sin ^{2} 2 b \tau$ in eq (II-1).

This factor has a maximum of unity for $b \tau=\pi / 4$. Since the pertur bation amplitude b is proportional to the square root of the applied power, the higher the applied microwave power, the higher is the velocity of the atoms for which the transition probability is maximum.

For the assumed fairly uniform velocity distribution the average velocity of the detected atoms is very close to the velocity where $\sin ^{2} b \tau$ is maximum and the linewidth can be calculated to a very good approximation by the relation

$$
W=\frac{1}{2 T_{a v}}=\frac{v_{a v}}{2 L}
$$


where $\mathrm{T}_{\mathrm{av}}$ and $\mathrm{v}_{\mathrm{av}}$ are the average time of flight and velocity, respectively. This is an approximation with an error of less than 3 percent even for the full modified Maxwellian, where

$$
\mathrm{W}=0.65 \frac{\alpha}{\mathrm{L}}=\frac{\mathrm{vav}}{2.05 \mathrm{~L}}
$$

If we now call $W_{0}$ the linewidth at the highest useful power $\mathrm{P}_{\mathrm{o}}$, the condition $\mathrm{b} \tau=\pi / 4=$ const. leads to the relation

$$
\frac{W}{W_{0}}=\sqrt{\frac{P_{0}}{P_{o}}} .
$$

Comparing this relation to figure 1 we see an exact fit at $\mathrm{P}=\mathrm{P}_{\mathrm{O}}$ and $\mathrm{P}=0.5 \mathrm{P}_{\mathrm{O}}$ and a very small error between these values. At very low power, our simple approximation is no longer valid.

Using eq (II-1) to calculate--as in Part I--the frequency $v_{1}$ to which an electronic servo using sinusoidal modulation locks leads to the simple result:

$$
\nu_{1}-\nu_{0}=\nu_{p}-\nu_{0}=\frac{W \varphi}{\pi}
$$

That there is no dependence on modulation amplitude is trivial in our simple case because of the symmetry of the line approximated by eq (II-1). Hellwig, et al., have shown that there is no significant dependence even for the full modified Maxwellian distribution. The total shift due to the phase difference $\varphi$ is $25 \%$ larger than that calculated for the full modified Maxwellian velocity distribution. This value appears to be more conservative for resonators having a restricted velocity distribution.

\section{CONCLUSIONS}

With the simple approximation of eq (II-1) derived directly from Ramsey's solution, we have thus been able to derive a relationship between power and linewidth which fits closely to the numerical data given in figure 1. We must point out again, however, that the expected variation of linewidth with excitation power is not observed 
in resonators having a narrow velocity distribution, or a sharp cutoff at the low velocity side of the distribution. There might be cases where there is a less sharp cutoff on the high velocity side and a correspondent line broadening could then be expected by applying higher than "optimum" power. Experimental investigation of this possibility is recommended. Results obtained with a commercial cesium beam tube are sijown in Table 1 .

\section{TABLE 1}

\begin{tabular}{|c|c|c|c|c|}
\hline $\mathrm{P} / \mathrm{P}_{\mathrm{O}}$ & $\left(\mathrm{P} / \mathrm{P}_{\mathrm{O}}\right)^{\frac{1}{2}}$ & $\mathrm{~W} / \mathrm{W}_{\mathrm{o}}$ & ${ }^{I / I_{\max }}$ & $\sin ^{2} 2 b \tau$ \\
\hline 0.5 & 0.707 & 0.971 & 0.80 & 0.80 \\
\hline 1 & 1 & 1 & 1 & 1 \\
\hline 2 & 1.414 & 1.17 & 0.638 & 0.632 \\
\hline
\end{tabular}

These results are consistent with the assumption of a very narrow velocity distribution. The variation of the linewidth as a function of excitation power is much smaller than that expected for a wide or full modified Maxwellian velocity distribution. The resonance amplitude behaves almost exactly as if only one single velocity were present. This behavior is indeed very different from that reported on the NBS-III cesium beam resonator in Part I.

\section{ACKNOWLEDGMENT}

The author would like to thank Dr. L. S. Cutler* and Mr. R. H. Kern ${ }^{* * *}$ for the communication and permission to use the results presented in Table 1.

\footnotetext{
*Hewlett-Packard Co., Palo Alto, California
} ***Frequency and Time Systems, Inc., Danvers, Massachusetts. 


\section{REFERENCES}

[1] Thirteenth General Conference of Weights and Measures (CGPM), Resolution l (1967).

[2] Glaze, D. J., "Improvements in atomic cesium beam frequency standards at the National Bureau of Standards, "IEEE Trans. Instrumentation and Mea surements, IM-19, No. 3, pp. 156-160 (August 1970).

[3] Ramsey, N. F., Molecular Beams, Clarendon Press, Oxford, England (1956).

[4] Mungall, A. G., "The second order Doppler shift in cesium atomic frequency standards," Metrologia, 7 , No. 2, pp. 49-56 (April 1971).

[5] Harrach, R. J., "Radiation-field-dependent frequency shifts of atomic beam resonances," J. Appl. Phys., 38, No. 4, pp. 18081819 (March 15, 1967).

[6] Becker, G., Fischer, B., Kramer, G., and Müller, E. K., "Neuentwicklung einer Cäsium-Strahl-Apparatur als primäres Zeit und Frequenz-Normal an der Physikalisch-Technische Bundesanstalt, " PTB-Mitteilungen, 79, pp. 77-80 (February 1969). 
FORM NBS-114A (1-71)

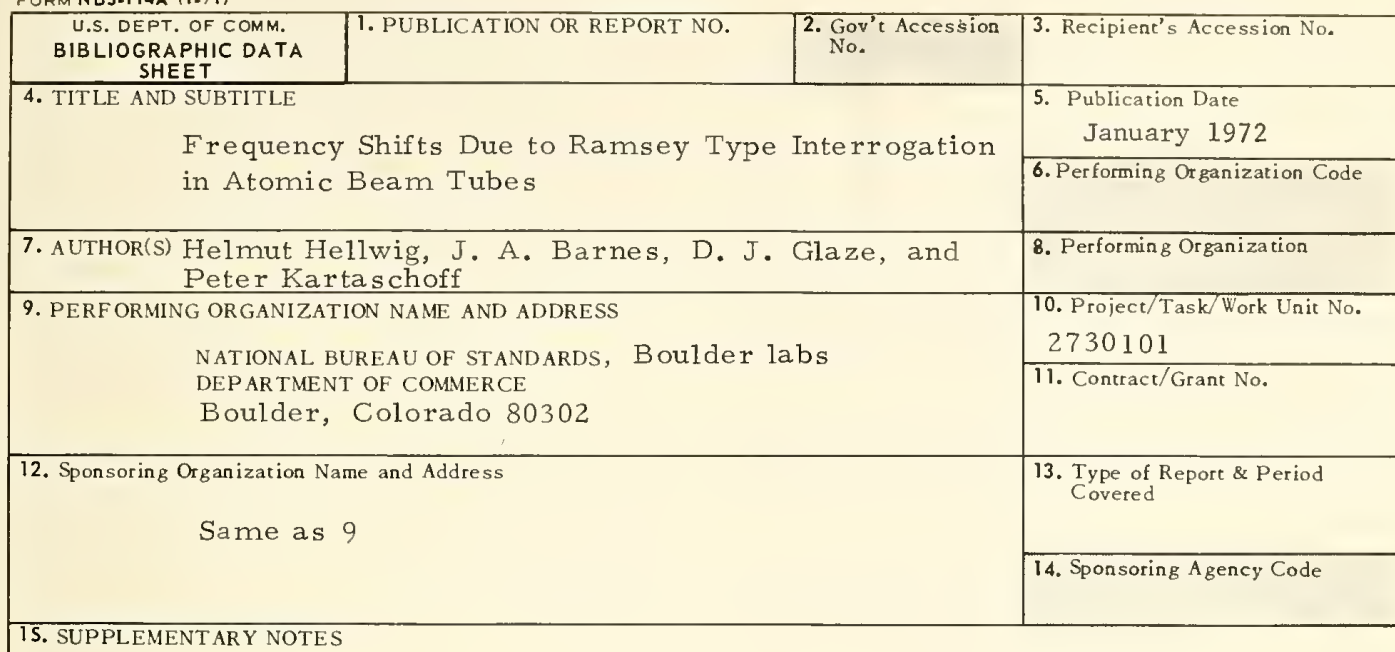

15. SUPPLEMENT ARY NOTES

16. ABSTRACT (A 200-word or Iess factual summary of most significant information. If document includes a significant bibliography or literature survey, mentionit here.) Part I: A phase difference between the two interaction regions of a Ramsey excitation resonance structure results in a frequency bias in the measured beam resonance. A simple mathematical model is discussed which describes the dependence of this bias on the phase difference, the microwave power level, the modulation amplitude, and the resonance linewidth. This dependence results from the interaction of the modulated microwave excitation frequency with the asymmetric shape of the slightly shifted resonance line. In a first order approximation, no dependency on the frequency modulation amplitude is expected. Near-linear dependencies on the linewidth and microwave power level which are quite pronounced even at relatively small cavity phase differences are predicted. The theoretical results are compared with one set of experimental data on the microwave power dependence as measured in 1969 with the primary cesium beam standard NBS-III. After a correction is applied to remove the power dependence due to spectral impurity of the microwave excitation, the remaining measured power dependence agrees quantitatively with that calculated using a cavity phase difference of about 3 milliradians.

Part II: One of the most serious effects limiting the accuracy of atomic beam resonato s is the uncertainty of phase shifts between the oscillating fields in a Ramsey excitation cavity. A technique using the linewidth dependence on the excitation power to measure the phase-shift induced bias has recently been proposed. The conditions under which this method is valid and their implications on the design of beam optics will be discussed with consideration of available experimental results.

17. KEY WORDS (Alphabetical order, separated by semicolons)

Cavity phase shift; cesium beam tube; frequency accuracy; frequency standard; power shift; resonance line shape.

\section{AVAILABILITY STATEMENT}

$\mathrm{X}$ UNLIMITED.

FOR OFFICIAL DISTRIBUTION. DO NOT RELEASE TO NTIS.

\begin{tabular}{|l|c|}
\hline $\begin{array}{l}\text { 19. SECURITY CLASS } \\
\text { (THIS REPORT) }\end{array}$ & 21. NO. OF PAGES \\
UNCL ASSIFIED & 25 \\
\hline $\begin{array}{l}\text { 20. SECUR ITY CLASS } \\
\text { (THIS PAGE) } \\
\text { UNCL ASSIFIED }\end{array}$ & 35 cents \\
\hline
\end{tabular}

USCOMM-DC $66244-P 71$ 

PERIODICALS

JOURNAL OF RESEARCH reports National Bureau of Standards research and development in physics, mathematics, chemistry, and engineering. Comprehensive scientific papers give complete details of the work, including laboratory data, experimental procedures, and theoretical and mathematical analyses. Illustrated with photographs, drawings, and charts.

Published in three sections, available separately:

\section{- Physics and Chemistry}

Papers of interest primarily to scientists working in these fields. This section covers a broad range of physical and chemical research, with major emphasis on standards of physical measurement, fundamental constants, and properties of matter. Issued six times a year. Annual subscription: Domestic, $\$ 9.50 ; \$ 2.25$ additional for foreign mailing.

\section{- Mathematical Sciences}

Studies and compilations designed mainly for the mathematician and theoretical physicist. Topics in mathematical statistics, theory of experiment design, numerical analysis, theoretical physics and chemisty, logical design and programming of computers and computer systems. Short numerical tables. Issued quarterly. Annual subscription: Domestic, \$5.00; $\$ 1.25$ additional for foreign mailing.

\section{- Engineering and Instrumentation}

Reporting results of interest chiefly to the engineer and the applied scientist. This section includes many of the new developments in instrumentation resulting from the Bureau's work in physical measurement, data processing, and development of test methods. It will also cover some of the work in acoustics, applied mechanics, building research, and cryogenic engineering. Issued quarterly. Annual subscription: Domestic, $\$ 5.00 ; \$ 1.25$ additional for foreign mailing.

\section{TECHNICAL NEWS BULLETIN}

The best single source of information concerning the Bureau's research, developmental, cooperative, and publication activities, this monthly publication is designed for the industry-oriented individual whose daily work involves intimate contact with science and technology-for enginecrs, chemists, physicists, research managers, product-development managers, and company executives. Annual subscription: Domestic, $\$ 3.00 ; \$ 1.00$ additional for foreign mailing.

\section{NONPERIODICALS}

Applied Mathematics Series. Mathematical tables. inanuals, and studies.

Building Science Series. Research results, test methods, and performance criteria of building materials, components, systems, and structures.

Handbooks. Recommended codes of engineering and industrial practice (including safety codes) developed in cooperation with interested industries, professional organizations, and regulatory bodies.

Special Publications. Proceedings of NBS conferences, bibliographies, annual reports, wall charts, pamphlets, etc.

Monographs. Major contributions to the technical literature on various subjects related to the Bureau's scientific and technical activities.

National Standard Reference Data Series.

NSRDS provides quantitative data on the physical and chemical properties of materials, compiled from the world's literature and critically evaluated.

Product Standards. Provide requirements for sizes, types, quality, and methods for testing various industrial products. These standards are developed cooperatively with interested Government and industry groups and provide the basis for common understanding of product characteristics for both buyers and sellers. Their use is voluntary.

Technical Notes. This series consists of communications and reports (covering both other agency and NBS-sponsored work) of limited or transitory interest.

Federal Information Processing Standards Publications. This series is the official publication within the Federal Government for information on standards adopted and promulgated under the Public Law 89-306, and Bureau of the Budget Circular A-86 entitled, Standardization of Data Elements and Codes in Data Systems.

Consumer Information Series. Practical information, based on NBS research and experience, covering areas of interest to the consumer. Easily understandable language and illustrations provide useful background knowledge for shopping in today's technological marketplace.

NBS Special Publication 305, Supplement 1, Publications of the NBS, 1968-1969. When ordering, include Catalog No. C13.10:305. Price \$4.50; $\$ 1.25$ additional for foreign mailing.

Order NBS publications from:

Superintendent of Documents

Government Printing Office

Washington, D.C. 20402 
National Bureau of Standards

Washington, 0.C. 20234

POSTAGE ANO FEES PAID

OFFICIAL BUSINESS

U.S. OEPAFTMENT OF COMMERCE

Penalty for Private Use, \$300

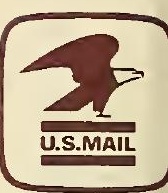






